\title{
An experimental test of deviant modeling
}

Owen Gallupe, $\mathrm{PhD}^{\mathrm{a}}$ (ogallupe@uwaterloo.ca)

Holly Nguyen, PhD ${ }^{\mathrm{b}}$ (hollynguyen@psu.edu)

Martin Bouchard, $\mathrm{PhD}^{\mathrm{c}}$ (mbouchard@sfu.ca)

Jennifer L. Schulenberg, PhDª (jlschule@uwaterloo.ca)

Allison Chenier, MA (achenier@uwaterloo.ca)

Katie D. Cook, MA ${ }^{\mathrm{a}}$ (k4cook@uwaterloo.ca)

${ }^{a}$ Department of Sociology and Legal Studies, University of Waterloo

${ }^{b}$ Department of Sociology and Criminology, Pennsylvania State University

${ }^{\mathrm{c}}$ School of Criminology, Simon Fraser University

Full citation:

Gallupe, O., Nguyen, H., Bouchard, M., Schulenberg, J., Chenier, A., \& Cook, K. D. (2016). An experimental test of deviant modeling. Journal of Research in Crime and Delinquency, 53(4), 482-505. DOI:10.1177/0022427815625093

\section{ACKNOWLEDGEMENTS}

This research was supported by a UW/SSHRC Seed Grant. The authors would like to thank Gowsigan Gowribalan, Joshua Laplante, Declan Smeaton, Rebecca Sim, and Manleen Singh for their research assistance and Abigail Scholer for her input on research design. 


\begin{abstract}
Objectives: Test the effect of deviant peer modeling on theft as conditioned by verbal support
\end{abstract} for theft and number of deviant models.

Methods: Two related randomized experiments in which participants were given a chance to steal a gift card (ostensibly worth \$15) from the table in front of them. Each experiment had a control group, a verbal prompting group in which confederate(s) endorsed stealing, a behavioral modeling group in which confederate(s) committed theft, and a verbal prompting plus behavioral modeling group in which confederate(s) did both. The first experiment used one confederate; the second experiment used two. The pooled sample consisted of 335 undergraduate students.

Results: Participants in the verbal prompting plus behavioral modeling group were most likely to steal followed by the behavioral modeling group. Interestingly, behavioral modeling was only influential when two confederates were present. There were no thefts in either the control or verbal prompting groups regardless of the number of confederates.

Conclusions: Behavioral modeling appears to be the key mechanism, though verbal support can strengthen the effect of behavioral modeling.

Keywords: Social learning theory; modeling; peer influence; experiment; theft. 
Scholars have long claimed that the relationship between peer deviance and personal deviance is among the strongest associations found in criminology (Warr 2002). In general, studies in this area show that individuals with higher levels of deviance tend to be those who a) associate with a greater number delinquent peers and/or peers who offend more frequently (e.g., Agnew 1991; Haynie 2001; Matsueda and Anderson 1998), b) associate with peers who exhibit higher levels of approval for deviance (e.g., Agnew 1991; Megens and Weerman 2012; Ragan 2014), and c) expect/receive stronger reinforcements favoring deviance (e.g., Akers, Krohn, Lanza-Kaduce, and Radosevich 1979; Brauer 2009). Much of the research has explicitly tested the propositions of Akers' (1998) Social Learning Theory (SLT) that delinquent contagion is a product of differential association, definitions in support of deviant behavior (i.e., holding values that support deviance), behavioral modeling/imitation, and differential reinforcement (i.e., deviance becomes more likely to the extent that others reward the act).

Despite the abundance of studies that examine deviant peer influence, few studies have focused on the modeling/imitation component of Akers' SLT; those that have done so have found the effect to be "modest at best" (Pratt et al. 2010:765). However, most studies that examine deviant peer influence are based on surveys which have inherent difficulties isolating the effect of modeling/imitation. Building on the work of Paternoster, McGloin, Nguyen, and Thomas (2013), we present an experimental test of the criminogenic effect of modeling.

\section{SOCIAL LEARNING AND MODELING}

According to Akers (1998:75), imitation is defined as "committing behavior modeled on, and following the observation of, similar behavior in others." In SLT, peer modeling of deviant behavior is thought to increase the likelihood that a person will engage in similar behavior (Akers 2009). As Sutherland (1947) notes in his Differential Association Theory (DAT), 
exposure to others who engage in illicit behavior provides the individual with examples of the techniques required to offend and the justifications for it. Both Sutherland and Akers note that the probability of imitation of the behavior is affected by the characteristics of the individual who models the behavior. For example, people who are well-liked and respected are more likely to be imitated (Cohen and Prinstein 2006). Further, imitation effects in general are influenced by the relative frequency, intensity, duration and priority of the association with deviant others. Neither Sutherland nor Akers, however, ruled out the possibility of imitation even if the individual modeling the behavior is not a member of the imitator's primary social group. For example, Akers (1998) specifically discussed the impact of the media on imitation of behavior and previous studies have demonstrated that confederates who displayed deviant behavior can be effective at eliciting imitation (e.g., Bandura 1965; Bandura, Ross, and Ross 1963; Gino, Ayal, and Ariely 2009; Lied and Marlatt 1979; Paternoster, McGloin, Nguyen, and Thomas 2013).

To situate our experiment within the existing peer influence literature, we will first address the ways that modeling has traditionally been measured in SLT research before focusing on the relatively small body of literature that has employed experimental methods.

\section{Traditional Approaches to Testing Deviant Modeling}

Studies that examine SLT tend to discuss modeling/imitation as being conceptually distinct from differential association. These concepts are, however, operationally very similar and past research has not been consistent in the types of measures used to assess them (see, for example, the different measurement approaches of Boeringer, Shehan, and Akers 1991; Cochran, Sellers, Wiesbrock, and Palacios 2011; Peralta and Steele 2010; Wareham, Boots, and Chavez 2009). While most research on SLT has employed survey methods (see the meta-analysis by Pratt et al. 2010 for a summary of this research), it is difficult to operationalize the behavioral 
modeling process in a way that does not overlap with differential association. This point is exemplified by Miller et al. (2011:312) who note that "measuring [modeling/imitation] remains elusive and difficult to isolate as the other components of social learning theory typically wash out the impact of imitation when measured concurrently." It is further supported by Fox, Nobles, and Akers (2011:43) who used what they refer to as a combined "differential association/modeling" scale.

The most common approach of peer influence research has been to ask survey participants to report their own levels of deviance and then to estimate the deviance of their friends. The correspondence between the two measures is then calculated (see, for example, Jensen 1972; Matsueda and Anderson 1998; Simons and Burt 2011; Warr and Stafford 1991). While much of the research characterizes peer associations with individuals involved in offending behavior as an umbrella measure of the peer effect that encompasses the various aspects of SLT, some survey-based SLT research has attempted to isolate the criminogenic effect of modeling by distinguishing between differential association and modeling/imitation. However, this distinction tends to be blurred. This reflects Akers' (1998) suggestion that differential association is an overarching conceptual category that includes modeling/imitation. ${ }^{1}$ Nonetheless, some studies discuss modeling/imitation specifically. For example, Akers et al. (1979:654) assessed imitation by creating a measure of the "total of all the "admired" models (parents, friends, other adults, etc.) whom the respondent reports having observed using [alcohol/marijuana]." ${ }^{2}$ They found that imitation had much less of a relationship to alcohol or marijuana use or abuse when compared to the differential association variables (number of friends the respondent reported to be an alcohol/marijuana user). 
Similarly, Krohn, Skinner, Massey, and Akers (1985) measured peer imitation of smoking by asking whether respondents' best male and female friends smoked. They found that imitation was only related to initiation of smoking through its influence on anticipated reinforcements. In other words, people with best friends who smoke are more likely to feel that their friends will support them for smoking and are therefore more likely to start. However, they characterized their model as "ineffective in explaining initiation of smoking" as the model only explained $3 \%$ of the variance (466).

While these studies and others (see, for example, Boeringer, Shehan, and Akers 1991; Peralta and Steele 2010; Wareham, Boots, and Chavez 2009) offer important insight into the relationship between modeling and imitation of deviant behavior, the researchers were unable to isolate a modeling effect. In order to facilitate this, the conceptual distinction between differential association and modeling must be clear. Essentially, differential association can be taken to mean having affiliations with people who offend but it does not necessarily require that they witness the offending behavior. Modeling/imitation involves more direct observation. Experimental methods might offer advantages over survey methods in terms of isolating the effect of modeling as the extent to which participants witness deviant behavior can be systematically manipulated by researchers. It is to this issue that we now turn. Experimental Studies of Deviant Modeling

McGloin and Thomas (2013) lamented the lack of experimental tests of criminological theory in general and noted that learning perspectives translate particularly well into experimental methods. The logic of experimental designs leads to the conclusion that any changes/differences in the outcome must be caused by differences in experimental treatment. Some experimental research has examined the influence of deviant models on individual 
behavior. For example, Bandura's (e.g., 1965; Bandura, Ross, and Ross 1963) seminal "Bobo doll" studies found that children exposed to adults who modeled physical and verbal aggression towards the doll were consequently more likely to exhibit aggression in their play, particularly if the modeler was rewarded for their behavior. Gardner and Steinberg (2005) found that the mere presence of peers increased the amount of risk-taking behavior exhibited by participants in a driving simulation though the effect was weaker among older participants.

Other research has taken advantage of roommate assignments of first year university students to emulate the randomization process. For example, Duncan, Boisjoly, Kremer, Levy, and Eccles (2005) found that males with a history of binge drinking tend to drink more if assigned a roommate who also binge drank in high school than if their roommate did not binge drink in high school. However, these peer influence effects on alcohol use did not occur for females or marijuana use for either males or females. One issue with studies employing roommate assignment designs is that they cannot rule out the possibility of shared experiences accounting for similarity in roommate behavior.

One way to reduce the possibility that shared experiences might account for peer similarity is to employ laboratory-based experiments. They may be limited in terms of external validity, but they allow researchers greater control over exogenous factors and are therefore useful in isolating specific dynamics. Some lab experiments have examined peer effects on alcohol use and related behavior. Such studies have found that peer modeling of alcohol use is related to higher levels of alcohol use (e.g., Collins, Parks, and Marlatt 1985; Lied and Marlatt 1979) and that people exposed to attitudes supporting drunk driving were more likely than others to drive dangerously in a simulator (Oei and Kerschbaumer 1990). The results from these studies suggest that peer modeling can have an effect on an individual's alcohol-related behavior but 
given high levels of acceptance of drinking behavior (Mears, Ploeger, and Warr 1998), alcohol use dynamics may differ from other forms of deviance.

The recent study by Paternoster et al. (2013) is among the first laboratory-based peer influence experiments in criminology and provides the foundation for the current study. In their study, participants were recruited on the basis that they were going to be involved in a memory study and would receive \$20 for their time. Experimental sessions were conducted in groups of between six and nineteen participants in a computer lab. Upon arrival, they were read a list of twenty words to recall later in the study and informed that they would receive $\$ 1$ for each word correctly recalled (all received $\$ 20$ in the end). When explaining the procedure to enter the recalled words into the computer, the experimenter acted surprised to find links that were not supposed to be there. Clicking on the links revealed that they contained the words to be recalled. The experimenter then left the room in order to fix the problem while participants were to be completing the memory task. He told participants to ignore the links. Once the experimenter left the room, the experimental treatment was presented (or not) by a research confederate. In the control group, the confederate did nothing out of the ordinary. In the treatment group, the confederate made it verbally known that he was taking the illegitimate route to improve his payout and then clicked on the links to reveal the words. These actions were visible to participants if they were looking. In both conditions the opportunity to illegitimately gain funds was equal. Paternoster et al. found that not a single participant in the control group cheated. However, $38 \%$ of participants in the experimental group cheated.

\section{CURRENT STUDY}

In order to examine the criminogenic effect of deviant peer modeling, we conducted two related randomized experiments that build on the work of Paternoster et al. (2013) in three ways. 
The first adjustment is in the type of outcome we examined. In our experiments, the possible deviant behavior was the theft of gift cards to local businesses. To steal the card, the participant had to reach out and take a card in front of others in the group. This meant that the deviant act was more visible than in the Paternoster et al. study. Further, our goal was to increase the seriousness of the deviant outcome beyond research that has focused on dishonesty as a way to improve performance-based remuneration (e.g., Gino, Ayal, and Ariely 2009; Paternoster et al. 2012).

The second adjustment is in the number and type of experimental groups that were included. Since the Paternoster et al. (2013) study did not distinguish between deviant affirmations (i.e., verbal modeling) and actual displays of deviance, we attempted to test the individual and combined effects of verbal and behavior modeling. Four groups (including a control group) were therefore used.

a) The Control Group received no cues.

b) The Verbal Prompting Group received verbal cues from the confederate supporting theft.

c) In the Behavioral Modeling Group, the confederate stole without saying anything.

d) In the Verbal Prompting plus Behavioral Modeling Group, the confederate showed support for theft verbally and stole.

The third adjustment is that we manipulated the number of confederates (separate experiments with one versus two confederates) to see if this conditioned the effect of modeling. It may be that the deviance of a single other person can be ignored while being exposed to multiple deviant models suggests that the behavior is normative and therefore more acceptable.

\section{METHODS}




\section{Sample}

The sample for both experiments consisted of undergraduate students 18 years of age and older from a moderate-to-large Canadian university (total $n=335$ ). Table 1 provides basic demographic information. Participants were mostly in their late teens/early 20s, over two thirds were female, and slightly less than half were white. There were no significant differences in terms of demographic characteristics between experimental groups. Experimental sessions were conducted between October 2013 and April 2014. Recruitment was done via email and posters distributed widely across campus advertising a study examining the sight of rewards on task performance. In the recruitment material, it was noted that participants would receive a $\$ 15$ gift card to a local business.

\section{[Insert Table 1 about here]}

\section{Procedure}

\section{Study 1.}

The first experiment $(n=168)$ tested the effect of modeling with a single confederate demonstrating the deviant behavior (or not). Upon contacting the research assistant to participate, respondents were randomized into one of the four groups and the assistant worked with the potential participant to find an appropriate time slot. Therefore, while the participant had some flexibility in terms of when they attended, this did not alter the randomization process (e.g., a person who was randomized into group 1 could select from a number of different time slots in which group 1 was scheduled to run).

Participants were told that their task was to unscramble as many of the 30 possible words as they could within the allotted 10 minutes. They were also told that the goal of the study was to see if people who could see rewards in front of them performed better on average than those who 
could not see the rewards. To support this, many gift cards were spread across the table in front of the participants who were seated at a table facing each other. These cards were ostensibly worth $\$ 15$ each though in reality they had no cash value. Participants were told that half of the groups had the cards placed in front of them while the other half did not. The gift cards were actually placed on the table for all groups. This was necessary to ensure all groups had the same opportunity to take the gift cards, which is crucial to the measurement of the dependent variable (individual deviant behavior).

The sessions started with the research assistant reading the information and consent forms to the participants. They were informed that they would receive 50 cents for each word correctly unscrambled. When the researcher reached this point, the research confederate interrupted and said "Wait, I thought we were getting \$15 regardless." The researcher responded "No, but you can make up to that." This was done to make participants feel slightly cheated with the idea that this would incentivize them to steal since theft was expected to be infrequent across all experimental groups and respondents were more likely to do so if they felt cheated. ${ }^{3}$ This closely mimicked the approach of Paternoster et al. (2013). After the initial consent process, the word scramble was distributed. It was intentionally very difficult to ensure that most people did not earn the full $\$ 15$. The average number of words correctly unscrambled in the allotted 10 minutes was 8.5 meaning that the typical respondent would be under the impression that they earned $\$ 4.25$, less than one third of the advertised amount $(\mathrm{SD}=4.0$ words, median $=8$, maximum $=22$; $50 \%$ of the sample correctly unscrambled between 6 and 11 words).

Once the word scramble was finished, the researcher collected the sheets, distributed the questionnaires, and announced that while participants were completing the questionnaire, she was briefly going to her office just down the hall to grade the word scrambles and get the correct 
remuneration amount in varying denominations of gift cards and change as required. The researcher was out of the lab for about 2 minutes. It was when the researcher left the room that the experimental conditions were applied. In the control group, the confederate did nothing other than the interruption during the information and consent stage. In the verbal prompting group, the confederate offered verbal support for theft but did not actually steal. In this condition, the confederate modeled the fact that they hold definitions that favor offending behavior. When the researcher left the room, the confederate said "This sucks. There's no way I did well enough to make \$15. I say we just grab one.” In the behavioral modeling condition, the confederate simply said "okay" to draw attention and then took a gift card from the table without giving verbal support for theft. Therefore, they modeled the deviant behavior strictly through their actions. In the verbal plus behavioral condition, the confederate made the same statement as in the verbal prompting group but also took a gift card from the table. The confederate therefore modeled the deviant behavior through their actions as well as displayed their definitions in support of it.

\section{Study 2.}

Our second randomized experiment $(n=167)$ included two confederates in each session to test whether the number of deviant models conditioned the effect of verbal prompting and behavioral modeling on the likelihood of stealing. ${ }^{4}$ The basic idea is that the effect of peers is likely to be stronger when there are more of them modeling the behavior. The procedures for the second experiment closely followed those of the first experiment but with minor changes to the script to incorporate the second confederate in three of the groups (in the control group, neither confederate did anything out of the ordinary aside from one confederate interrupting the researcher at the information and consent stage). In the verbal prompting condition, the following banter between the two confederates was scripted: 
Confederate 1: "This sucks. There's no way I did well enough to make $\$ 15 . "$

Confederate 2: "Ya, this is bullshit. We were definitely promised $\$ 15 . "$

Confederate 1: "I wouldn’t have come if I knew I was only getting like \$5."

Confederate 2: (Pointing at the cards) "Fuck it. We should all grab one."

In the behavioral modeling group, the first confederate said "okay" and took a gift card from the table. The second confederate laughed and also took a gift card. For the verbal plus behavioral modeling group, a minor adaptation to the end of the script was implemented as piloting showed that it came across unnaturally to end the verbal exchange at the same spot as in the verbal prompting group. The confederates stated the following and then both took a gift card:

Confederate 1: "This sucks. There's no way I did well enough to make $\$ 15$."

Confederate 2: "Ya, this is bullshit. We were definitely promised $\$ 15$."

Confederate 1: "I wouldn’t have come if I knew I was only getting like \$5."

Confederate 2: (Pointing at the cards) "Fuck it. Grab one."

Confederate 1: "And get ratted out?"

Confederate 2: "Who's going to say anything?"

Confederate 1: "True. I'm in if you are."

Confederate 2: "Done."

The confederates in both experiments were young adult males. Participants were debriefed immediately after the session. This happened in one of two ways. For sessions in which nobody stole a card, participants were debriefed together given the low likelihood of participants being upset. ${ }^{5}$ However, in sessions where a participant took a gift card, debriefing was done individually for all participants and in no particular order so as not to single out anyone. All participants received a $\$ 15$ gift card to a local business (either a coffee shop, grocery 
store, or the campus retail store) after debriefing. This study received ethics clearance from the review board at the first author's university and no adverse events occurred. ${ }^{6}$

\section{Treatment dilution}

Some treatment dilution occurred in this study where participants who were randomized into a treatment group ended up in the control group (Loughran and Mulvey 2010). In a small number of sessions, participants started conversing before the confederates began their script. In these situations, the confederates were instructed to default to the control group as it would appear unnatural to interrupt the discussion to apply an experimental treatment. There are essentially two possible approaches to handling treatment dilution (Gartin 1995). The first is to include them in the treatment groups that were actually received (e.g., Berk and Sherman 1985). The second is to employ the intention-to-treat principle and include cases in the groups they were originally randomized into regardless of whether dilution occurred. The intention-to-treat approach is more appropriate when assessing the implementation of a policy in which inconsistent application of the policy is to be expected. In the present study, there is no policy implementation being tested and the focus is clearly on the effect of the treatment as received, not treatment as intended. For this reason, we included dilution cases in the experimental groups that participants actually experienced regardless of the groups they were initially randomized into. That is, the analyses were conducted with all dilution cases in the control group as that is what they experienced. Table 2 shows the number of participants in each experimental group.

[Insert Table 2 about here]

\section{Variables}

The outcome variable is whether or not a participant took a gift card from the table when the researcher left the room. Each seat in the experimental session was labeled so that the 
confederate(s) could note the seat numbers of people who took a gift card. These seat identifiers were used to match theft reports to the participants' questionnaire. The predictor variable is the experimental group: (1) control, (2) verbal prompting, (3) behavioral modeling, (4) verbal prompting plus behavioral modeling.

\section{RESULTS}

The results of each experiment will first be presented separately before pooling them into a single analysis. In the one confederate experiment, only 2\% (4 out of 168) of participants took a gift card (see table 3). All of these occurred in the verbal prompting plus behavioral modeling group where $10 \%$ (4 out of 39) of the sample stole (see table 4). Differences between groups in the prevalence of theft was statistically significant (Fisher's exact $p=.005$; low expected cell counts ruled out the use of a Pearson chi-square test).

[Insert Table 3 about here]

[Insert Table 4 about here]

A significantly greater proportion of participants took a gift card in the two confederate experiment ( $7 \%-12$ out of 167$)$ than in the one confederate experiment (2\%) (Fisher's exact $p=$ .043 - see table 3). In both the behavioral modeling and verbal prompting plus behavioral modeling groups, $15 \%$ (6 out of 39) took a card (see table 4). Between-group differences were statistically significant (Fisher's exact $p<.001$ ).

Given the similarity between the two studies, we pooled the samples and tested them together. Approximately $5 \%$ of participants in the combined sample (16 out of 335) took a gift card. This low prevalence was expected given the relatively high value of the theft ( $\$ 15$ per gift card taken) and the fact that taking a card would mean doing so in full view of other participants. There were no thefts in either the control group or the verbal prompting group. However, $8 \%$ of 
participants in the behavioral modeling group (6 out of 73 ) and $13 \%$ in the verbal prompting plus behavioral modeling group (10 out of 78$)$ took a gift card. These differences were statistically significant (Fisher's exact $p<.001$ ). These results suggest that behavioral modeling of theft influences the likelihood of individual theft. However, the criminogenic effect of behavioral modeling is enhanced when combined with verbal support for theft.

The shaded portion of table 4 shows how the effect of modeling varied according to the number of confederates in the session. Nobody in the control group stole regardless of the number of confederates. Further, providing verbal support for theft had no influence (in the absence of behavioral modeling) as no thefts occurred in either the one or two confederate verbal prompting groups. What is particularly interesting is that when only a single confederate behaviorally modeled theft (without any verbal prompting), no thefts occurred. However, when two confederates behaviorally modeled theft, $15 \%(n=6$ out of 39$)$ of participants took a gift card. This is the same percentage of participants who stole in the two confederate verbal plus behavioral group. With a single confederate, theft only occurred in the verbal plus behavioral group (10\% - 4 out of 39). These differences were statistically significant (Fisher's exact $p<$ $.001)^{7}$

\section{DISCUSSION}

Over two decades ago, Warr and Stafford (1991:851) observed that "although the association between delinquent friends and delinquent behavior is well established, the mechanism by which delinquency is socially transmitted remains unclear." It is certainly the case that advances in our understanding of peer influence dynamics have been made in the interim, however their observation is just as valid now as it was then. Studies that examine deviant peer influence have overwhelmingly supported the claim that peer deviance is one of the most robust 
predictors of delinquency, regardless of method (survey, longitudinal, network, quasiexperimental, experimental). But there are still gaps in our knowledge of how they matter. In this study, we experimentally tested the criminogenic influence of modeling, something for which previous survey research has found only modest support.

By conducting two closely related experiments, we found the criminogenic effect of deviant modeling to be quite strong. Being around peers who steal was found to increase the likelihood of individual theft. The effect was enhanced when more peers engaged in theft and also when peers provided verbal support for stealing. Our results differ from the meta-analysis by Pratt et al. (2010). They aggregated the results of 133 social learning studies published between 1974 and 2003 and found that the criminogenic effect of modeling tended to be quite weak. A possible explanation for the difference between the present study and previous studies that rely on surveys is that reports of peer involvement in offending behavior is often based on imperfect knowledge (e.g., rumor). People may be less likely to follow the behaviors of their peers if they are not entirely certain that their peers actually engaged in the behavior. But a person may be more likely to imitate their peers if they know for sure that they committed a particular act.

Compared to the traditional approach of asking survey respondents to estimate the offending of their peers, social network studies claim to provide more objective measures as they utilize self-reports of peer offending (e.g., Haynie 2001). However, neither approach necessarily involves the individual directly witnessing peers engaging in these behaviors and therefore they rely on assumptions that the individual both knows about and believes reports of the behaviors of those around them. When presenting participants with models that they see actually committing a deviant act, the effect was found to be quite robust. 
Further, having more peers who model deviant behavior makes it more likely that the individual will follow in their footsteps. Although not the primary focus of the current study, models of collective behavior have examined the number or proportion of individuals one requires to engage in a particular behavior (e.g., Granovetter 1978; Haynie 2002; McGloin and Rowan 2015). According to Granovetter (1978), individuals vary in the threshold level of peer group involvement required before they are willing to commit a given act. One avenue of future research is to examine the threshold effect in a laboratory setting. Where the deviance of a single peer might be easy to ignore, it is possible that having multiple others in the immediate environment engaging in deviant behavior suggests that it is the desirable course of action. Further, where multiple deviant peers can spur behavior by their actions alone, we find that a single deviant peer can be influential if they both physically display the behavior and provide verbal support for it. The implication appears to be that, when compared with multiple peers who can simply do the act, a single deviant peer must exert more effort to have an influence.

Another potential reason for the difference between the results of the present study and most previous survey-based peer influence research is that the notion of influence is difficult to measure given the multifaceted and overlapping nature of learning mechanisms. It can therefore be challenging to isolate the various sources of influence using questionnaires. The clearest way to get at these mechanisms may be through direct, experimentally manipulated observation.

Akers (1998) recognized the importance of interactive effects of peer influence (e.g., he noted that there is a greater probability of imitating observed behavior if positive reinforcements are also present). The results of our study suggest that a renewed interest in these effects is warranted. Two findings in our study illustrate the importance of being sensitive to moderation: a) the number of deviant peers is a critical component in determining the effect of deviant 
modeling; b) the criminogenic effect of behavioral modeling is enhanced by verbal support for offending. While previous research has examined interactions related to peer influence (e.g., Agnew 1991; Megens and Weerman 2012), little has been done in this area using experimental methods. Given the known difficulty in detecting interactions in non-experimental research (McClelland and Judd 1993), future tests of peer influence dynamics would do well to consider experimental approaches.

The current study was specifically intended to disentangle the modeling of deviant behavior from verbal affirmations. We therefore frame and interpret our results in terms of deviant modeling. We would be remiss, however, if we did not discuss alternative theoretical explanations. Specifically, the results of the experiment demonstrated that subjects who observed a confederate taking the gift card had a higher probability of taking the gift card themselves. As noted by an anonymous reviewer, this study may simply present participants with an opportunity to engage in a deviant act that is appropriate under the circumstances (e.g., Keizer, Lindenberg and Steg 2008) as opposed to addressing any sort of learning mechanism. However, the gift cards were visibly displayed on the table and subjects in all conditions were made aware of their presence by the researcher. The fact that there were differences in theft prevalence across the experimental groups despite the fact that the opportunity to do it was held constant suggests that the study tapped into factors beyond simply the opportunity to engage in offending behavior.

Furthermore, observing two confederates take gift cards might decrease perceptions about the likelihood of detection and therefore increase the probability of theft. ${ }^{8}$ While this may be true, Akers (2009: 209) noted that "imitation is predicated on observing or being aware of the behavior." The probability of imitating the modeled behavior is based on other factors such as characteristics of the person modeling the behavior and any observed consequences resulting 
from the behavior (see also Bandura, Ross and Ross, 1963). ${ }^{9}$ Akers and Jenson (2006) moreover argue that contextual and situational influences do not preclude social learning mechanisms such as imitation and reinforcement. Therefore, while seeing multiple other people steal might reduce perceptions of risk, peer behaviors are still being imitated. The important point is that despite the likely importance of other dynamics, this study highlights the influence of peer modeling on theft. We believe that disentangling the reasons for imitation is important and therefore future research might incorporate a qualitative component to a similar study to uncover the precise mechanisms behind why a theft occurred (or not).

Our push for more extensive use of experimental methods in criminological theory testing is due to the enhanced capacity to interpret results as being causal as opposed to correlational (this echoes the perspective of McGloin and Thomas 2013). However, general limitations of individual-level, laboratory-based experiments are well known. Samples tend to be smaller and it is generally not feasible to collect representative samples. Further, it is difficult to experimentally test mediating pathways and ethical concerns limit the ability to examine more serious offending behavior or manipulate important covariates such as socioeconomic status. Therefore, experiments are not the only worthwhile approach to peer influence research. As a field, criminology should continue to be "catholic" in its methods (Sampson 2010:490). That is, we should take the conservative approach by examining issues from multiple methodological angles and draw conclusions only when evidence from a variety of sources supports it. Instead of the two approaches being oppositional, "a healthier approach to criminological science is to proceed along a dual track of statistical and experimental research" (Sampson 2010:497). Within this approach, however, criminology as a field and peer influence research specifically would benefit from more widespread use of experimental methods. While Sampson notes that there has 
been a recent surge in the use of criminological experiments, peer influence research has not followed this trend despite being particularly amenable to it (McGloin and Thomas 2013). We hope that this study plays a role in spurring a more extensive experimental agenda to move research into SLT forward.

There are a number of specific limitations that should be kept in mind when interpreting the results of this study. The major one is the sample. An undergraduate sample is not representative of the general population and students from one university are not representative of university students as a whole. University students tend to be from the mid to upper socioeconomic strata and are therefore less subject to social class based risk factors for criminal behavior. But at the same time, lifestyles/routine activities of students tend to put them at risk for criminal victimization and involvement (Fisher, Sloan, Cullen, and Lu 1998). It would be useful to replicate this experiment using various age categories, social class indicators, racial/ethnic groups, and regions. Experimental methods capturing a broader swath of society than is possible with an undergraduate sample seems to be the best way to establish or refute the generality of SLT and criminogenic peer influence more broadly. It would also be informative to vary the type of outcome in future studies (e.g., gift cards of different values).

The second limitation is the context of the offence. We created a situation in which the potential to steal arose after ostensibly being cheated. In a way, some of the participants might have justified stealing the gift cards as an illegitimate way to right a wrong. This type of scenario is relevant when offending is a possible reaction to mistreatment or adverse conditions (i.e., strain-inducing situations or conditions of relative deprivation). It is unclear how the results of this study would generalize to contexts in which individuals are in an unprimed state and therefore we underscore the importance of extending this study to different circumstances. 
Finally, the uniformity in the confederates can be viewed as both a strength and weakness. Using the same confederates minimized the possibility of participants reacting differently to the experimental conditions based solely on characteristics of the confederate(s) and therefore reduced a potential source of error. However, these characteristics are likely to be important. Homophily (the tendency for people to prefer dealing with others who are similar to themselves) is a powerful force (McPherson, Smith-Lovin, and Cook 2001), but in-group versus out-group identities can also differentially influence deviant behavior (Gino, Ayal, and Ariely 2009). By varying characteristics of the confederate along age, race, sex and possibly other lines, we could draw more accurate conclusions regarding the nature of deviant peer influence.

In all, this study provides evidence that having more peers who model deviant behavior has a causal effect on individual deviance (theft). However, the effect of modeling varies depending upon specific characteristics of peer behavior. In particular, the experimental conditions reproducing a situation where participants were presented with verbal support for deviance was not conducive to theft (no matter how many peers support these definitions); neither was the behavioral modeling condition where a single peer was shown to steal without verbal prompting. Thefts occurred when two peers behaviorally modeled theft, and when both verbal and behavioral cues occurred at the same time regardless of how many deviant peers were present. These findings offer more support for the criminogenic effect of modeling than has been found in most previous research. 


\section{NOTES}

${ }^{1}$ It could be argued that this experiment is a test of differential association. However, as Akers (1998:62) notes, differential association largely subsumes the other SLT mechanisms. He is explicit on this point: "The groups with which one is in differential association provide the major social contexts in which all the mechanisms of social learning operate. Not only do they expose one to definitions, they present models to imitate and mediate differential reinforcement...for criminal or conforming behavior." Therefore, tests of SLT dynamics (such as our experiment on modeling) are inherently tests of differential association.

${ }^{2}$ As noted by an anonymous reviewer, this actually measures the observation of behavior which may or may not be imitated.

${ }^{3}$ While doing this meant that participants were subjected to a form of strain (Agnew 1992), this experiment is not a useful test of General Strain Theory as the type of strain was constant for all participants.

${ }^{4}$ Participant characteristics were similar in the two experiments. Bivariate analyses $(t$ tests, chisquare tests) showed that there were no significant differences between participants in the one versus two confederate experiments in terms of age, race, income, or grades though females were overrepresented in the two confederate experiment. These sex differences appear to be a product of chance.

${ }^{5}$ In fact, numerous participants mentioned a sense of self-satisfaction that conditions were created specifically to promote theft, but they resisted. This suggests that participants did not suspect the true objective of the experiment.

${ }^{6}$ Pilot sessions were conducted prior to commencing the study to ensure that the methods were believable and to train the research team. Piloting was done in two stages. The first stage 
involved graduate students who knew the procedure and goals of the study. Refinements based on feedback from the graduate student pilot session were then tested on a "genuine" sample of undergraduates who were recruited in the same way and subjected to the same procedures as the final sample $(\mathrm{n}=20)$.

${ }^{7}$ The pooled sample cannot be considered to be truly randomized since the participants who were in the one confederate experiment did not have the chance to experience conditions with two confederates and vice versa. We therefore ran a series of supplementary multivariate models to test the robustness of results (available on request). In these models, we controlled for age, sex, race, grades, income, the number of words correctly unscrambled, and the number of participants in the session. Firth logit models were used since there were zero counts in some of the experimental groups (Firth 1993; see also Heinze \& Schemper 2002). As a further test, we ran multilevel linear probability models specifying random intercepts across sessions. The results of the regression models supported the bivariate results.

${ }^{8}$ We thank our anonymous reviewers for pointing this out.

${ }^{9}$ There may be further reluctance to view our findings as support for modeling/imitation because of the short time span between presentation of deviant modeling and subsequent deviant imitation. However, Akers does not explicitly state that modeling/imitation is a long term process. In fact, Akers (1998) states that the effect of deviant models varies as a function of relative frequency, intensity, duration, and priority. This means that short term exposure can still exert an influence on behavior but that the influence is likely to be weaker than long term exposure to peer deviance. Akers also draws heavily on Bandura whose famous Bobo doll experiment included a relatively short time span between presentation of the aggressive model and children displaying aggression. 


\section{REFERENCES}

Agnew, Robert. 1991. "The interactive effects of peer variables on delinquency." Criminology 29:47-72.

Agnew, Robert. 1992. "Foundation for a general strain theory of crime and delinquency." Criminology 30:47-88.

Akers, Ronald L. 1998. Social learning and social structure: A general theory of crime and deviance. Boston: Northeastern University Press.

-. 2009. Social learning and social structure: A general theory of crime and deviance. Brunswick, N.J.: Transaction.

Akers, Ronald L. and Gary F. Jensen. 2006. "The empirical status of social learning theory of crime and deviance: The past, present, and future.” Pp. 37-76 in Taking stock: The status of criminological theory, edited by F. T. Cullen, J. P. Wright, and K. R. Blevins. New Brunswick, NJ: Transaction Publishers.

Akers, Ronald L., Marvin D. Krohn, Lonn Lanza-Kaduce, and Marcia Radosevich. 1979. "Social learning and deviant behavior: A specific test of a general theory." American Sociological Review 44:636-655.

Bandura, Albert. 1965. "Influence of models' reinforcement contingencies on the acquisition of imitative responses." Journal of Personality and Social Psychology 1:589-595.

Bandura, Albert, Dorothea Ross, and Sheila A. Ross. 1963. "Vicarious reinforcement and imitative learning." Journal of Abnormal and Social Psychology 67:601-607.

Berk, Richard A. and Lawrence W. Sherman. 1985. "Data collection strategies in the Minneapolis domestic assault experiment." Pp. 35-48 in Collecting evaluation data: 
Problems and solutions, edited by L. Burstein, H. E. Freeman, and P. H. Rossi. Beverly Hills, CA: Sage.

Boeringer, Scot B., Constance L. Shehan, and Ronald L. Akers. 1991. "Social contexts and social learning in sexual coercion and aggression: Assessing the contribution of fraternity membership." Family Relations 40:58-64.

Brauer, Jonathan R. 2009. "Testing social learning theory using reinforcement's residue: A multilevel analysis of self-reported theft and marijuana use in the National Youth Survey." Criminology 47:929-970.

Cochran, John K., Christine S. Sellers, Valerie Wiesbrock, and Wilson R. Palacios. 2011. "Repetitive intimate partner victimization: An exploratory application of social learning theory." Deviant Behavior 32:790-817.

Cohen, Geoffrey L. and Mitchell J. Prinstein. 2006. "Peer contagion of aggression and health risk behavior among adolescent males: An experimental investigation of effects on public conduct and private attitudes." Child Development 77:967-983.

Collins, R. Lorraine, George A. Parks, and G. Alan Marlatt. 1985. "Social determinants of alcohol consumption: the effects of social interaction and model status on the selfadministration of alcohol." Journal of Consulting and Clinical Psychology 53:189-200.

Duncan, Greg J., Johanne Boisjoly, Michael Kremer, Dan M. Levy, and Jacque Eccles. 2005. "Peer effects in drug use and sex among college students." Journal of Abnormal Child Psychology 33:375-385.

Firth, David. 1993. "Bias reduction of maximum likelihood estimates." Biometrika 80:27-38.

Fisher, Bonnie S., John J. Sloan, Francis T. Cullen, and Chunmeng Lu. 1998. "Crime in the ivory tower: The level and sources of student victimization." Criminology 36:671-710. 
Fox, Kathleen A., Matt R. Nobles, and Ronald L. Akers. 2011. "Is stalking a learned phenomenon? An empirical test of social learning theory." Journal of Criminal Justice 39:39-47.

Gardner, Margo and Laurence Steinberg. 2005. "Peer influence on risk taking, risk preference, and risky decision making in adolescence and adulthood: An experimental study." Developmental Psychology 41:625-635.

Gartin, Patrick R. 1995. "Dealing with design failures in randomized field experiments: Analytic issues regarding the evaluation of treatment effects." Journal of Research in Crime and Delinquency 32:425-445.

Gino, Francesca, Shahar Ayal, and Dan Ariely. 2009. "Contagion and differentiation in unethical behavior: The effect of one bad apple on the barrel." Psychological Science 20:393-398.

Granovetter, Mark. 1978. "Threshold models of collective behavior." American Journal of Sociology 83:1420-1443.

Haynie, Dana L. 2001. "Delinquent peers revisited: Does network structure matter?" American Journal of Sociology 106:1013-1057.

—. 2002. "Friendship networks and delinquency: The relative nature of peer delinquency." Journal of Quantitative Criminology 18:99-134.

Heinze, Georg and Michael Schemper. 2002. "A solution to the problem of separation in logistic regression." Statistics in Medicine 21:2409-2419.

Jensen, Gary F. 1972. "Parents, peers, and delinquent action: A test of the differential association perspective." American Journal of Sociology 78:562-575.

Keizer, Kees, Siegwart Lindenberg, and Linda Steg. 2008. "The spreading of disorder." Science 322:1681-1685. 
Krohn, Marvin D., William F. Skinner, James L. Massey, and Ronald L. Akers. 1985. "Social learning theory and adolescent cigarette smoking: A longitudinal study." Social Problems 32:455-473.

Lied, Erik R. and G. Alan Marlatt. 1979. "Modeling as a determinant of alcohol consumption: Effect of subject sex and prior drinking history." Addictive Behaviors 4:47-54.

Loughran, Thomas A. and Edward P. Mulvey. 2010. "Estimating treatment effects: Matching quantification to the question." Pp. 163-180 in Handbook of Quantitative Criminology, edited by A. R. Piquero and D. Weisburd. New York: Springer.

Matsueda, Ross L. and Kathleen Anderson. 1998. "The dynamics of delinquent peers and delinquent behavior." Criminology 36:269-308.

McClelland, Gary H. and Charles M. Judd. 1993. "Statistical difficulties of detecting interactions and moderator effects." Psychological Bulletin 114:376-390.

McGloin, Jean, and Zachary R. Rowan. 2015. "A threshold model of collective crime." Criminology 53:484-512.

McGloin, Jean and Kyle Thomas. 2013. "Experimental tests of criminological theory." Pp. 15-42 in Experimental Criminology: Prospects for Advancing Science and Public Policy, edited by B. C. Welsh, A. A. Braga, and G. J. N. Bruinsma. New York: Cambridge University Press.

McPherson, Miller, Lynn Smith-Lovin, and James M. Cook. 2001. "Birds of a Feather: Homophily in Social Networks." Annual Review of Sociology 27:415-444.

Mears, Daniel P., Matthew Ploeger, and Mark Warr. 1998. "Explaining the gender gap in delinquency: Peer influence and moral evaluations of behavior." Journal of Research in Crime and Delinquency 35:251-266. 
Megens, Kim C. I. M. and Frank M. Weerman. 2012. "The social transmission of delinquency: Effects of peer attitudes and behavior revisited." Journal of Research in Crime and Delinquency 49:420-443.

Miller, Bryan Lee, John M. Stogner, David N. Khey, Ronald L. Akers, John Boman, and O. Hayden Griffin. 2011. "Magic Mint, the Internet, and Peer Associations: A Test of Social Learning Theory Using Patterns of Salvia Divinorum Use." Journal of Drug Issues 41:305-325.

Oei, Tian P. S. and D. M. Kerschbaumer. 1990. "Peer attitudes, sex, and the effects of alcohol on simulated driving performance." The American journal of drug and alcohol abuse $16: 135-146$.

Paternoster, Ray, Jean Marie McGloin, Holly Nguyen, and Kyle J. Thomas. 2013. "The causal impact of exposure to deviant peers: An experimental investigation." Journal of Research in Crime and Delinquency 50:476-503.

Peralta, Robert L. and Jennifer L. Steele. 2010. "Nonmedical prescription drug use among US college students at a Midwest university: a partial test of social learning theory." Substance use \& misuse 45:865-887.

Pratt, Travis C., Francis T. Cullen, Christine S. Sellers, L. Thomas Winfree, Jr., Tamara D. Madensen, Leah E. Daigle, Noelle E. Fearn, and Jacinta M. Gau. 2010. "The empirical status of social learning theory: A meta-analysis." Justice Quarterly 27:765-802.

Ragan, Daniel T. 2014. "Revisiting "what they think": Adolescent drinking and the importance of peer beliefs." Criminology.

Sampson, Robert J. 2010. "Gold standard myths: Observations on the experimental turn in quantitative criminology." Journal of Quantitative Criminology 26:489-500. 
Simons, Ronald L. and Callie Harbin Burt. 2011. "Learning to be bad: Adverse social conditions, social schemas, and crime." Criminology 49:553-597.

Sutherland, Edwin H. 1947. Principles of Criminology, vol. 4. Philadelphia: J. B. Lippincott.

Wareham, Jennifer, Denise Paquette Boots, and Jorge M. Chavez. 2009. "A test of social learning and intergenerational transmission among batterers." Journal of Criminal Justice $37: 163-173$.

Warr, Mark. 2002. Companions in crime. New York: Cambridge University Press.

Warr, Mark and Mark Stafford. 1991. "The influence of delinquent peers: What they think or what they do?" Criminology 29:851-866. 
Table 1. Descriptive Statistics

\begin{tabular}{|c|c|c|c|c|c|}
\hline & Mean & SD & Min & Max & \\
\hline Age & 20.50 & 3.04 & 18 & 52 & \\
\hline Male $(=1)$ & 0.31 & 0.46 & 0 & 1 & \\
\hline White $(=1)$ & 0.48 & 0.50 & 0 & 1 & \\
\hline Means & Control & $\begin{array}{c}\text { Verbal } \\
\text { Prompting }\end{array}$ & $\begin{array}{c}\text { Behavioral } \\
\text { modeling }\end{array}$ & $\begin{array}{c}\text { Verbal + } \\
\text { behav }^{\text {a }}\end{array}$ & $p$ \\
\hline \multicolumn{6}{|l|}{ Pooled sample } \\
\hline Age & 20.35 & 20.79 & 20.23 & 20.71 & $.624^{\mathrm{b}}$ \\
\hline Male $(=1)$ & 32.74 & 23.19 & 32.88 & 34.62 & $.439^{\mathrm{c}}$ \\
\hline White $(=1)$ & 56.52 & 49.28 & 38.36 & 42.31 & $.068^{\mathrm{c}}$ \\
\hline \multicolumn{6}{|c|}{ One confederate } \\
\hline Age & 20.35 & 20.77 & 19.94 & 19.79 & $.542^{\mathrm{b}}$ \\
\hline Male $(=1)$ & 39.68 & 26.67 & 44.12 & 43.59 & $.450^{\mathrm{c}}$ \\
\hline White $(=1)$ & 56.92 & 53.33 & 38.24 & 48.72 & $.353^{\mathrm{c}}$ \\
\hline \multicolumn{6}{|c|}{ Two confederate } \\
\hline Age & 20.35 & 20.79 & 20.49 & 21.62 & $.231^{\mathrm{b}}$ \\
\hline Male $(=1)$ & 24.00 & 20.51 & 23.08 & 25.64 & $.959^{\mathrm{c}}$ \\
\hline White $(=1)$ & 56.00 & 46.15 & 38.46 & 35.90 & $.217^{\mathrm{c}}$ \\
\hline
\end{tabular}


Table 2. Number of Participants per Experiment

\begin{tabular}{lccc}
\hline & \multicolumn{2}{c}{ Number of confederates } & \\
& One & Two & Total \\
\hline Control & 65 & 50 & 115 \\
Verbal & 30 & 39 & 69 \\
Behavioral & 34 & 39 & 73 \\
Verbal + behavioral & 39 & 39 & 78 \\
\hline Total & 168 & 167 & 335 \\
\hline
\end{tabular}


Table 3. Thefts by Number of Confederates $-\%$ (n)

\begin{tabular}{lccc}
\hline & \multicolumn{2}{c}{ Number of confederates } & \\
& One & Two & Total \\
\hline No theft & $97.62(164)$ & $92.81(155)$ & $95.22(319)$ \\
Theft & $2.38(4)$ & $7.19(12)$ & $4.78(16)$ \\
Total & $100(168)$ & $100(167)$ & $100(335)$ \\
\hline
\end{tabular}

Fisher's exact $p=.043$ 
Table 4 . Thefts by Condition - \% (n)

\begin{tabular}{lccc}
\hline & \multicolumn{2}{c}{ Experiment } & \\
& One confederate & Two confederate & Pooled sample \\
\hline Control & $0(0)$ & $0(0)$ & $0(0)$ \\
Verbal prompting & $0(0)$ & $0(0)$ & $0(0)$ \\
Behavioral modeling & $0(0)$ & $15.38(6)$ & $8.22(6)$ \\
Verbal + behav $^{\mathrm{a}}$ & $10.26(4)$ & $15.38(6)$ & $12.82(10)$ \\
$p$ & $.005^{\mathrm{b}}$ & $<.001^{\mathrm{c}}$ & $<.001^{\mathrm{d}}$ \\
\hline
\end{tabular}

Note: Values are the percentage ( $\mathrm{n}$ in brackets) of participants in a particular cell that took a gift card. E.g., 10\% of participants in the one confederate verbal prompting plus behavioral modeling group took a gift card.

Fisher's exact test for differences across the eight groups in the shaded portion: $p<.001$.

${ }^{a}$ Verbal prompting plus behavioral modeling group.

${ }^{b}$ Fisher's exact test for differences across the four groups for those in the one confederate experiment only.

'Fisher's exact test for differences across the four groups for those in the two confederate experiment only.

'Fisher's exact test for differences across the four groups for those in the pooled sample (both one and two confederate experiments). 